\title{
Identification and functional analysis of bull (Bos taurus) cauda epididymal fluid proteome
}

\author{
B. Westfalewicz, ${ }^{* 1}$ M. A. Dietrich, ${ }^{*}$ A. Mostek, ${ }^{*}$ A. Partyka, $†$ W. Bielas, $†$ W. Niżański, $†$ and A. Ciereszko* \\ *Department of Gamete and Embryo Biology, Institute of Animal Reproduction and Food Research, Polish Academy of Sciences, Tuwima 10, \\ 10-748 Olsztyn, Poland \\ †Department of Reproduction and Clinic of Farm Animals, Faculty of Veterinary Medicine, Wrocław University of Environmental and Life Sciences, \\ pl. Grunwaldzki 49, 50-366 Wrocław, Poland
}

\section{ABSTRACT}

Despite recent advances in bull epididymal fluid proteome research, significant numbers of proteins secreted to epididymal lumen remain unidentified. The objective of this study was to expand the number of identified cauda epididymal fluid proteins in bulls and to contextualize them in a broader view of their mutual interactions and involvement in biological processes and pathways, to fully elucidate the ways in which epididymal fluid proteins are involved in storage and maturation of spermatozoa in epididymis. We collected postmortem cauda epididymal fluid from 6 mature Holstein Friesian bulls. We performed the identification of proteins using 2-dimensional electrophoresis coupled with MALDI mass spectrometry. Analysis of functionality and pathway involvement of identified proteins was performed using Ingenuity Pathway Analysis software. We identified a total of 189 epididymal fluid proteins, out of which 100 were newly identified in bull epididymal fluid. We have combined our data with 2 previously performed bull epididymal fluid proteome identifications, yielding 280 proteins total, and analyzed it. The main canonical pathways involving epididymal proteins were glycolysis, gluconeogenesis, protein ubiquitination pathway, nuclear factor-erythroid 2-related factor 2-mediated oxidative stress response, and farnesoid X receptor/retinoid $\mathrm{X}$ receptor activation. The main biological functions potentially performed by epididymal fluid proteins included carbohydrate metabolism, cellular growth and proliferation, cell death and survival, and small molecule biochemistry. Overall, our results have pointed out multiple novel pathways in bull epididymal fluid that might take part in various aspects of maturation and protection processes of epididymal spermatozoa.

Key words: bull, epididymis, proteome

Received December 27, 2016.

Accepted March 30, 2017.

${ }^{1}$ Corresponding author: b.westfalewicz@pan.olsztyn.pl

\section{INTRODUCTION}

The epididymis is a complex, convoluted tube that connects efferent ducts to the vas deferens in the male reproductive tract (Robaire et al., 2006; Dacheux et al., 2016). The mammalian epididymis usually consists of 3 regions: the initial segment, the head (or caput), the body (or corpus), and the tail (or cauda). The most basic function of the epididymis is the transportation of spermatozoa, delivering them from testicles to lower parts of the male reproductive tract. The epididymis also serves as effective storage for male gametes, preserving the viability of spermatozoa for up to $2 \mathrm{wk}$ in the case of the bull. During transportation and storage, the absorptive and secretory activities of the epididymal epithelium create a specialized luminal environment that allows for sperm maturation. Epididymal sperm maturation is the final step of postgonadal sperm differentiation, during which spermatozoa acquire functional maturity manifested by development of fertilizing ability and motility. After ejaculation, mature sperm suspended in epididymal fluid is mixed with remaining secretions of male reproductive tract, in the case of the bull mainly seminal vesicle fluid, creating semen (Juyena and Stelletta, 2012).

Recent advances in electrophoresis, MS, and bioinformatics have allowed for a breakthrough in the comprehensive identification of mammalian semen proteins. Such an approach is called proteomics, which is research of the proteome (protein complement of the genome; Wilkins et al., 1996). Research of the bull reproductive tract proteome has led to the description of numerous proteins of seminal plasma and reproductive tract secretions (Kelly et al., 2006; Moura et al., 2007; Westfalewicz et al., 2017). This knowledge could be useful for cattle breeding for improving fertility or performance in sample storage (Soggiu et al., 2013; De Canio et al., 2014). Recently, we were able to describe the seminal vesicle fluid proteome and its functional similarity to seminal plasma proteome for the first time (Westfalewicz et al., 2017). Strong focus was directed 
to find proteins connected with fertility localized in spermatozoa (D'Amours et al., 2010), seminal plasma (Odhiambo and Dailey, 2011), and epididymal fluid (Moura et al., 2006).

The first major work identifying bull epididymal secretion proteome was performed by Moura et al. (2010), who identified 30 proteins of cauda epididymal fluid. Belleannée et al. (2011) improved on this work and identified 172 luminal and secreted proteins from every region of the epididymis. Those works gave insight into the possible functionality of proteins identified and provided important information on changes occurring in the epididymal fluid of successive epididymal regions. Despite those advances, a significant part of the bull epididymal fluid proteome remains unexplored, as evidenced by several thousand proteins identified in epididymal secretions of more thoroughly investigated species such as humans or rats (Dacheux et al., 2016). Moreover, the description of proteins identified in bull epididymal fluid has been focused mainly on singular proteins and their evidenced or putative functions in the epididymis, with more comprehensive analysis being limited to rough functional classification of proteins using Gene Ontology tools. For this reason, we believe that further comprehensive research focused on mutual protein relationships, functions of protein groups, and protein involvement in metabolic pathways is justified.

In this study, we identified 100 new cauda epididymal fluid proteins and combined our results with those of previous epididymal fluid proteome identifications to obtain the most robust data set possible, consisting of 280 proteins. This combined data set was then analyzed for protein functions and metabolic pathways, to better understand bull epididymal fluid proteome functionality.

\section{MATERIALS AND METHODS}

\section{Sample Collection}

Samples were collected from six 4-yr-old Holstein Friesian bulls. The bulls were provided by the Breeding and Insemination Station in Karczew, Poland. Cauda epididymal fluid was collected postmortem from the bulls by the Department of Reproduction and Clinic of Farm Animals (Wrocław, Poland). The testicles of each bull were collected within 3 min after slaughter and transported at $4^{\circ} \mathrm{C}$ to the laboratory. In the laboratory, epididymides were dissected from the testes. The surface of each epididymis was thoroughly cleaned from the remnants of tissue and blood vessels and washed with physiological saline $(0.9 \% \mathrm{NaCl})$. After removing the sodium chloride, the tail of the epididymis was punctured by an incision needle and the epididymal fluid containing spermatozoa was expelled by gentle pressure. Collection of the fluid was finished when the liquid changed color (from white to light pink). No additional fluids (buffers or PBS) were used for the liquid collection. Epididymal fluid was centrifuged for $30 \mathrm{~min}$ at $4^{\circ} \mathrm{C}(900 \times g)$. Next, the supernatant was centrifuged for $10 \mathrm{~min}$ at $4^{\circ} \mathrm{C}(3,000 \times g)$. After collection, the epididymal fluid was checked microscopically for the presence of blood cells. Samples with blood cells present were discarded. Supernatant was then stored at $-80^{\circ} \mathrm{C}$ until the time of further studies.

\section{Sample Preparation for 2-Dimensional SDS-PAGE}

After thawing, samples of epididymal fluid $(\mathrm{n}=$ 6) were centrifuged for $60 \mathrm{~min}$ at $4^{\circ} \mathrm{C}(10,000 \times g)$ to remove any remaining debris from the liquid. After centrifugation, aliquots containing approximately $700 \mu \mathrm{g}$ of protein were cleaned with a Clean-Up Kit (GE Healthcare, Uppsala, Sweden) according to the manufacturer's protocol (https://www.gelifesciences. com/gehcls_images/GELS/Related\%20Content/ Files/1314735988470/litdoc80648964_20161013181035. pdf). The protein concentration before and after the cleaning procedure was measured by the Coomassie (Bradford) Protein Assay Kit (Thermo Fisher Scientific, Waltham, MA).

\section{2-Dimensional SDS-PAGE}

Samples of cauda epididymal fluid containing 500 $\mu \mathrm{g}$ of protein were resuspended in rehydration buffer (7 $M$ urea, $2 M$ thiourea, $2 \%$ \{3-[(3-cholamidopropyl)dimethylammonio]-1-propane-sulfonate\}, $2 \%$ immobilized $\mathrm{pH}$ gradient buffer, $40 \mathrm{~m} M$ dithiothreitol, $0.002 \%$ bromophenol blue) to reach a final volume of $450 \mu \mathrm{L}$. Each sample was then loaded onto 24-cm Immobiline DryStrips, 3 to 10 nonlinear $\mathrm{pH}$ range (GE Healthcare), and rehydrated for $10 \mathrm{~h}$. Proteins were then separated by isoelectric focusing on an Ettan IPGphor apparatus (GE Healthcare) operating at $20^{\circ} \mathrm{C}$ with current limited to $50 \mu \mathrm{A}$ per strip and the following voltage program: $500 \mathrm{~V} / 5 \mathrm{~h}, 1,000 \mathrm{~V} / 1 \mathrm{~h}, 8,000 \mathrm{~V} / 3 \mathrm{~h}$, and $8,000 \mathrm{~V} / 5.5$ $\mathrm{h}$. After isoelectric focusing, the strips were equilibrated for $15 \mathrm{~min}$ in SDS equilibration buffer (6 $M$ urea, 75 $\mathrm{m} M$ Tris- $\mathrm{HCl}, \mathrm{pH} 8.8,29.3 \%$ glycerol, $2 \%$ SDS, and a trace of bromophenol blue) containing $10 \mathrm{mg} / \mathrm{mL}$ of dithiothreitol and then for $15 \mathrm{~min}$ in SDS equilibration buffer containing $25 \mathrm{mg} / \mathrm{mL}$ of iodoacetamide. The equilibrated strips were then transferred to manually cast $12.5 \%$ gels $(25.5 \times 19.6 \mathrm{~cm}, 1 \mathrm{~mm}$ thickness $)$ and sealed with $0.5 \%$ agarose. A second dimension of electrophoresis was then performed at $1 \mathrm{~W} /$ gel in an Ettan Dalt-Six apparatus (GE Healthcare) for $16 \mathrm{~h}$. 


\section{Image Acquisition and Analysis}

The electrophoretic gels of 6 individual cauda epididymal fluid proteome were stained using Coomassie Brilliant Blue G250 and scanned with an ImageScanner III (GE Healthcare). All acquired images were analyzed separately using an Image Master 2D Platinum 7.0 (GE Healthcare). The software was used to automatically detect the protein spots in each gel. Spots consistently occurring in images from every individual were selected for identification. Selected spots were indicated for identification in a spot pick list created by the software.

Protein spot volume was calculated by the Image Master 2D Platinum software by normalizing the volume of each spot from each gel against total spot volume. All proteins above the value of $1 \%$ of total spot volume were considered to be major proteins.

\section{Excision and Spot Identification Using MALDI-TOF/TOF}

Spots were automatically picked from gels with an Ettan Spot Picker (GE Healthcare), which picked the spots on the basis of a spot pick list generated by Image Master Platinum. Picked spots were put in a 96-well collection plate and transferred to an Ettan Digester (GE Healthcare). Spots were then digested using the following protocol: (1) 3 consecutive washes with $200 \mu \mathrm{L}$

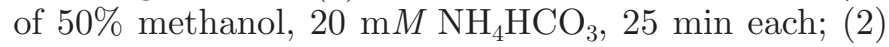
2 consecutive washes with $200 \mu \mathrm{L}$ of $100 \%$ acetonitrile, 5 min each; (3) $100 \mu \mathrm{L}$ of wash with $100 \%$ acetonitrile, left to dry for over $15 \mathrm{~min}$; and (4) the addition of 10 $\mu \mathrm{L}$ of $0.2 \mu \mathrm{g} / \mu \mathrm{L}$ of modified sequencing grade trypsin

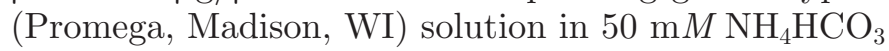
followed by incubation for $12 \mathrm{~h}$ at $37^{\circ} \mathrm{C}$. Peptide mixtures with gel spots were then sonicated briefly with a VCX-130 Ultrasonic Processor (Sonics \& Materials Inc., Newtown, CT), put in a Speedvac Concentrator (Thermo Fisher Scientific), and dried in a vacuum. The dried gel spots were discarded. Dried samples were then placed in an Ettan Spotter robot (GE Healthcare), where they were dissolved in matrix solution ( $5 \mathrm{mg}$ of

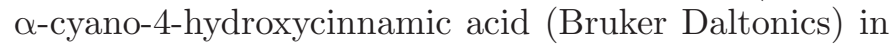
$1 \mathrm{~mL}$ of $50 \%$ acetonitrile in $0.1 \%$ trifluoroacetic acid) and spotted onto the MALDI target plate (MT 34 Target Plate Ground Steel, Bruker Daltonics) using the method "Dissolve Sample in Matrix First - Then Spot" with the following parameters: matrix volume, $3 \mu \mathrm{L}$; mix cycles, number 5; mix volume, $3 \mu \mathrm{L}$; spot volume, $0.9 \mu \mathrm{L}$; and airgap, $3 \mu \mathrm{L}$. After spotting, samples were left to dry. The MALDI-TOF/TOF MS analysis was carried out using a MALDI-TOF tandem mass spectrometer (Autoflex Speed, Bruker Daltonics). Collected
MS and MS/MS LIFT spectra of selected ions were externally calibrated using monoisotopic $(\mathrm{MpH}) \mathrm{b}$ ion peptide calibration standards (Bruker Daltonics) and imported to BioTools (Bruker Daltonics). The data were searched using a Mascot Server (Matrix Science, London, UK) in the National Center for Biotechnology Information database, with the following parameters: fixed modifications: carbamidomethyl (C); variable modifications: oxidation; cleavage enzyme: trypsin; maximum missed cleavages: 2; peptide mass tolerance: $100 \mathrm{mg} / \mathrm{kg}$; and fragment mass tolerance: $0.6 \mathrm{kDa}$ (Perkins et al., 1999). Mascot database search provided the statistical significance of protein scores and peptide ion scores, determined by the software. Proteins were defined as identified when the protein score, along with at least 2 peptide ion scores, was statistically significant $(P<0.05)$. When a protein was occurring in multiple proteoforms, one of them had to be identified with at least 2 significant peptide ion scores, and remaining proteoforms could be successfully identified with at least one significant peptide ion score. Protein spots unidentified in the first attempt were cut, digested, and analyzed again. Additionally, to validate the identification, we selected 225 spots and identified them again (Supplemental Table S1; https://doi.org/10.3168/ jds.2016-12526).

\section{Ontology and Ingenuity Pathway Analyses of the Identified Proteins}

Gene Ontology annotation was acquired by matching the GenInfo Identifier numbers of identified proteins to the UniProtKB database (www.uniprot.org; Huntley et al., 2015). The categories used were "molecular functions" and "biological processes." Ingenuity Pathway Analysis (IPA; Qiagen Silicon Valley, Redwood City, $\mathrm{CA}$ ) of the identified proteins used the "core analysis" function to interpret the identified proteins in the context of biological functions and pathways. The IPA was performed on epididymal fluid proteins identified by our work, supplemented with data from Moura et al. (2010) and Belleannée et al. (2011). Three proteins were excluded from the analysis, as they were found by Belleannée et al. (2011) exclusively in the caput region of the epididymis, and our work has focused on cauda epididymal fluid. We also removed the "colanic acid building blocks biosynthesis" canonical pathway from the list of top 20 canonical pathways, as we were unable to find evidence of its existence in mammals. Part of this removed pathway, however, was shared with the GDP-mannose biosynthesis (GMB) pathway. The significance of biological functions and canonical pathways was tested automatically by the software, with use of the Fisher exact test $P$-value. 


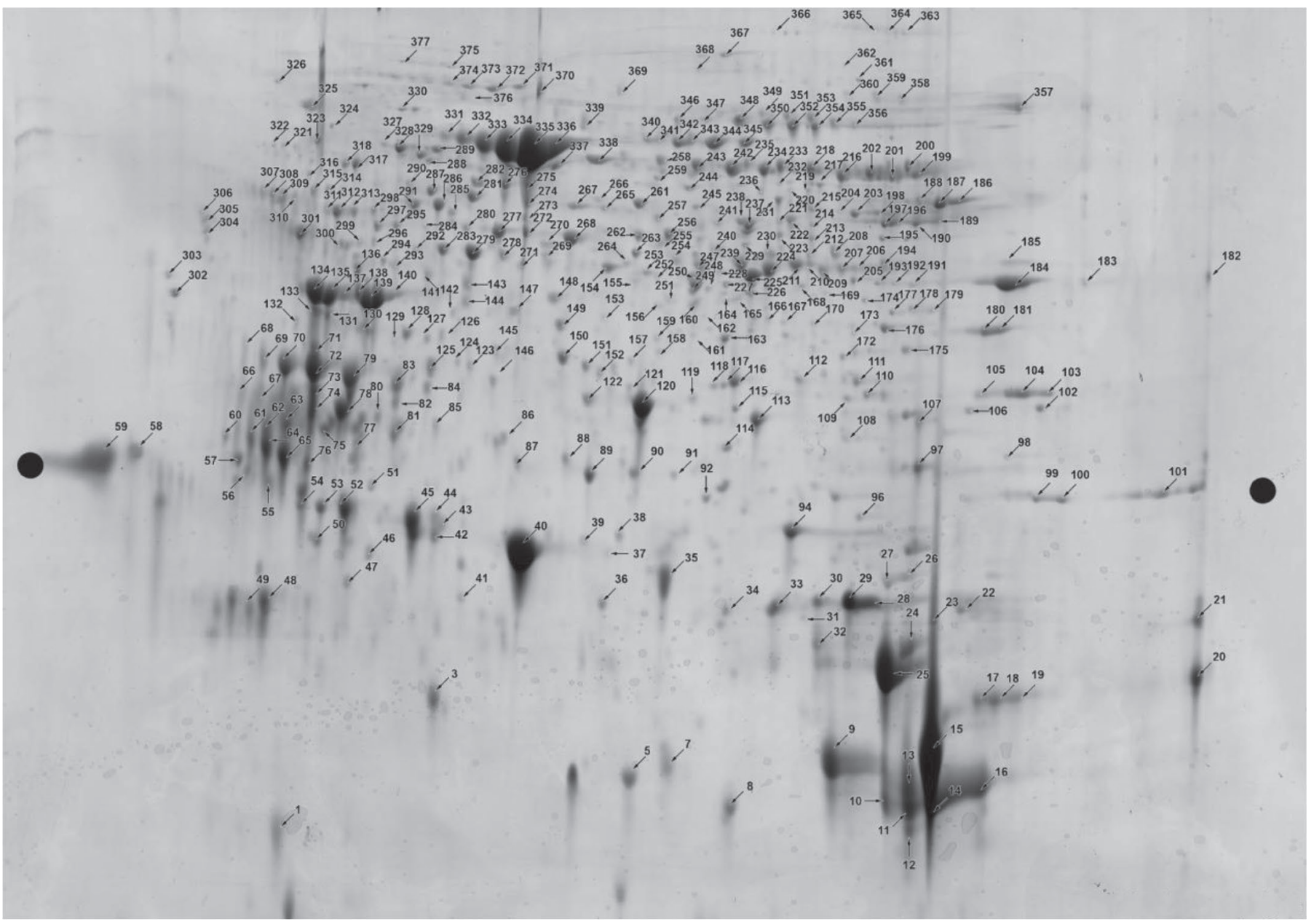

Figure 1. Two-dimensional SDS-PAGE gel of bull cauda epididymal fluid; numbered arrows correspond to spot numbers presented in Supplemental Table S2 (https://doi.org/10.3168/jds.2016-12526).

\section{RESULTS}

\section{Characteristic of Bull Epididymal Fluid Proteome}

Analysis of 2-dimensional SDS-PAGE gels of individual cauda epididymal fluid collected from 6 bulls allowed for detection of an average of $546(\mathrm{SD}=72$, $\mathrm{CV}=0.13$ ) spots on each gel (Figure 1). Spots consistently occurring in images from every individual were selected for identification. Three hundred sixty-seven protein spots found in all 6 gels/bulls were identified, corresponding to 189 different proteins. The full list of proteins is presented in Supplemental Table S2 (https://doi.org/10.3168/jds.2016-12526). Nine proteins were over the total spot volume of $1 \%$ (Figure 2 ). Epididymis-specific estrogen receptor $\alpha$ binding protein-like protein, represented by 8 spots, accounted for the highest percentage of total spot volume detected in gels $(19.63 \%)$, followed by clusterin represented by
20 spots $(8.44 \%)$, serum albumin precursor represented by 11 spots (7.04\%), WAP 4-disulfide core domain protein 2 precursor represented by 2 spots $(3.8 \%)$, hexosaminidase B represented by 13 spots $(2.69 \%)$, serotransferrin precursor represented by 9 spots $(2.63 \%)$, prostaglandin-H2 D-isomerase precursor represented by 4 spots (2.52\%), aldose reductase represented by 3 spots $(1.42 \%)$, and cytoplasmic actin 1 represented by 4 spots $(1.22 \%)$. Together, all these protein spots accounted for $49.39 \%$ of total spot volume detected in the gel.

\section{Ingenuity Pathway Analysis}

We combined proteins identified in our work with those identified by Moura et al. (2010) and Belleannée et al. (2011), which allowed us to analyze a total of 280 proteins. Functional analysis of identified epididymal fluid proteins by IPA allowed for detection of functions and canonical pathways associated with proteins. The 
top 20 canonical pathways and biological functions of epididymal proteins are presented in Tables 1 and 2, respectively. Full information on canonical pathways and biological functions of bull cauda epididymal proteins is presented in Supplemental Tables S3 and S4 (https:// doi.org/10.3168/jds.2016-12526).

\section{Gene Ontology Analysis}

Identified epididymal fluid proteins were also subjected to Gene Ontology analysis (Figure 3). Of 280 proteins, 245 were successfully categorized according to molecular function and biological process. Based on molecular function analysis, proteins were divided into 9 groups, with the majority having binding and hydrolase activity. Based on biological process analysis, proteins were divided into 11 groups, with the majority being involved in cellular processes, metabolic processes, and biological regulation.

\section{DISCUSSION}

In this study, we expanded significantly the number of identified proteins in bull cauda epididymal fluid. Our work resulted in identification of 189 proteins, of which 100 were previously not found in bull epididymal fluid. By adding proteins identified by our work to those identified by Moura et al. (2010) and Belleannée et al. (2011), we acquired the most complete epididymal fluid proteome to date, containing 280 proteins, which allowed comprehensive functional analysis to unravel the biological role of epididymal proteins (Figure 4).

Analysis of our results suggests that carbohydrate metabolism is an important part of epididymal physiology. This suggestion is supported by the fact that of the top 20 canonical pathways, 5 were involved in carbohydrate metabolism: glycolysis, gluconeogenesis, sucrose degradation, pentose phosphate (PP) pathway, and GMB. Glycolysis is a vital part of semen activity,

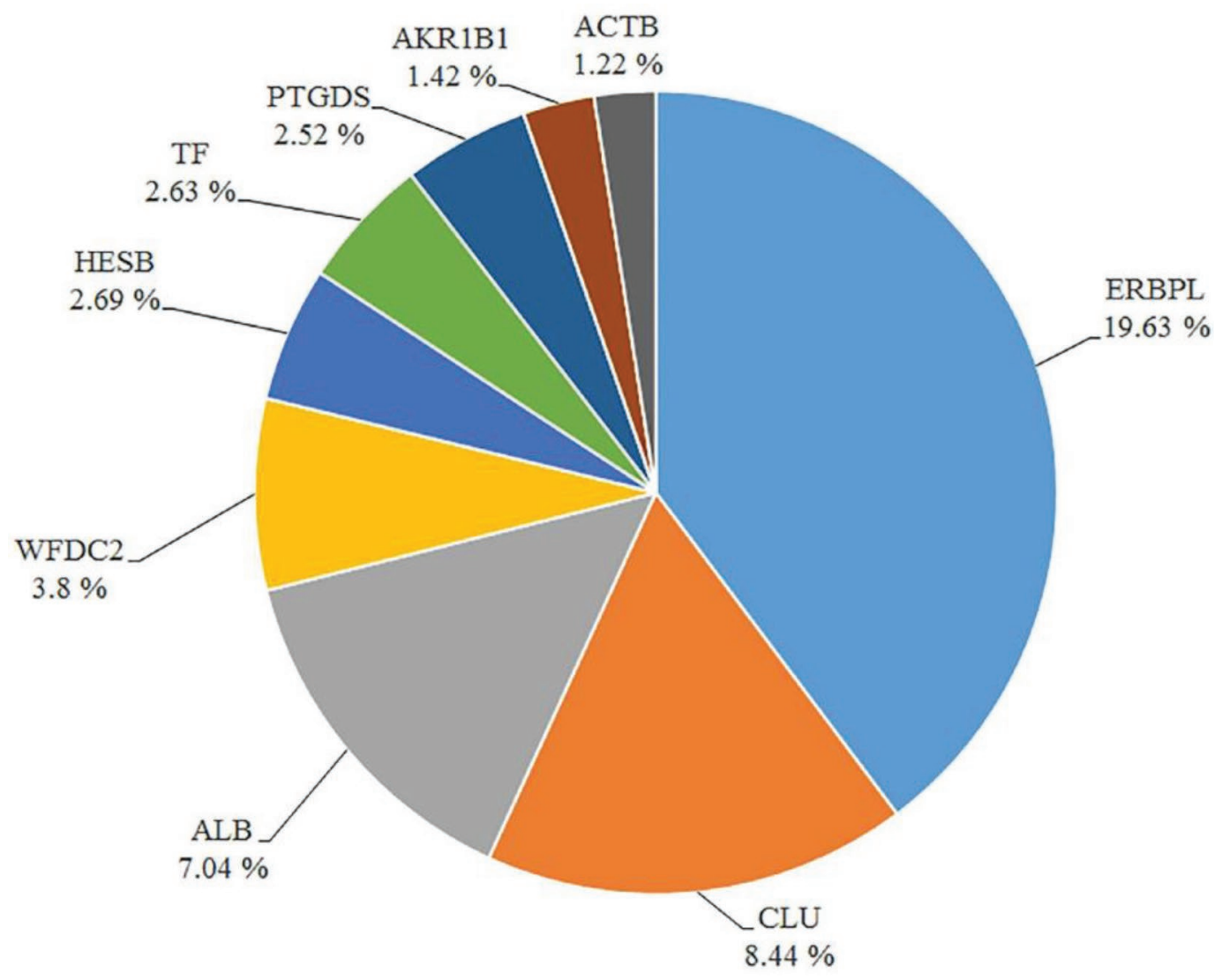

Figure 2. Pie chart presenting the major proteins of bull cauda epididymal fluid, as indicated by the analysis of volume of spots visible in the electropherogram presented in Figure 1. All major proteins accounted for $49.39 \%$ total spot volume. ERBPL = epididymis-specific estrogen receptor $\alpha$ binding protein-like protein; CLU = clusterin; ALB = serum albumin precursor; WFDC2 $=$ WAP 4 -disulfide core domain protein 2 precursor; HESB = hexosaminidase $\mathrm{B} ; \mathrm{TF}=$ serotransferrin precursor; PTGDS = prostaglandin-H2 D-isomerase precursor; AKR1B1 = aldose reductase; $\mathrm{ACTB}=$ cytoplasmic actin 1 . Color version available online. 


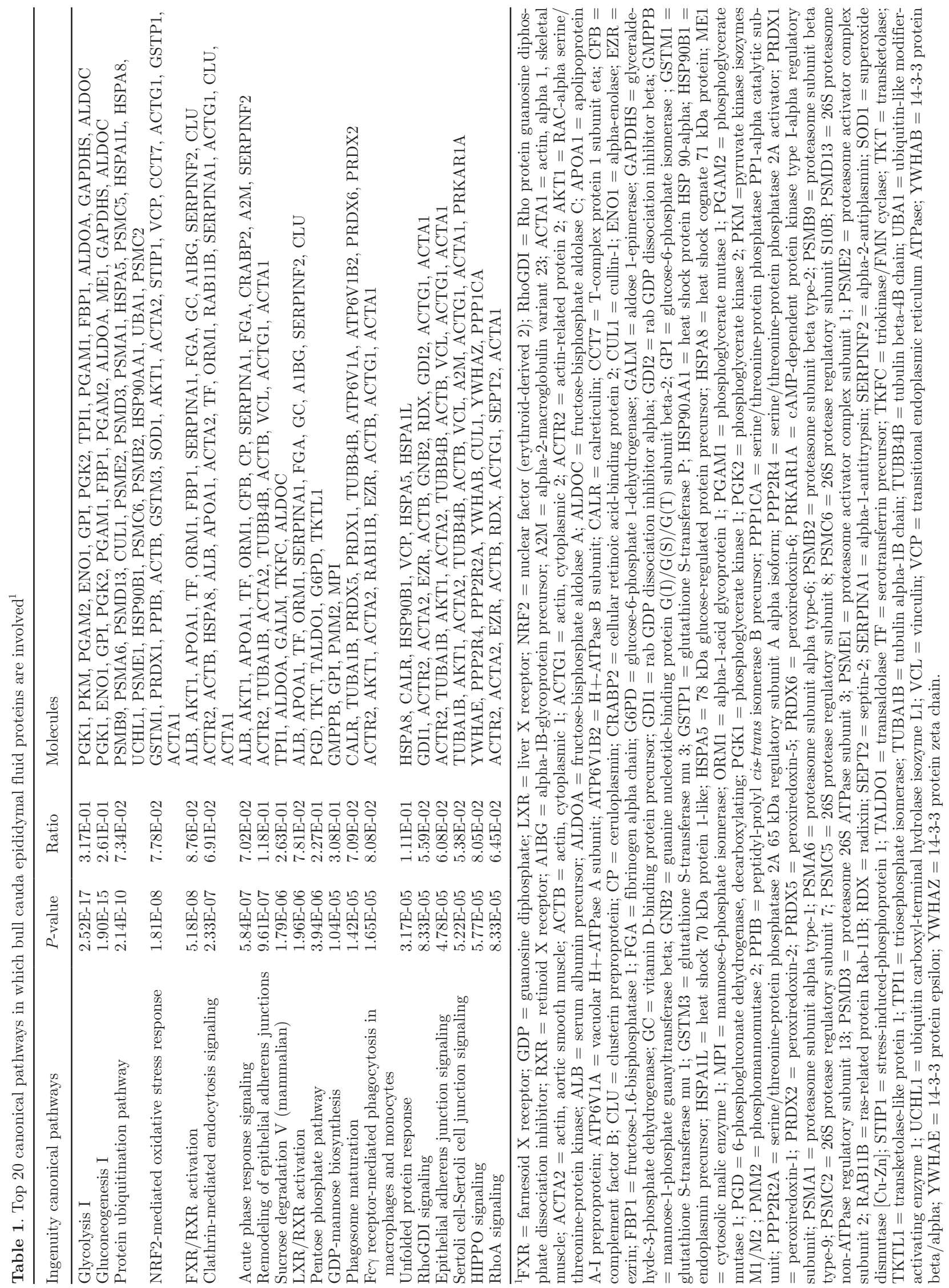


as it is involved in capacitation, provides a secondary source of energy for sperm cells, and is closely related to spermatozoa functional maturity and epididymal transit (Hoskins et al., 1975; Panse et al., 1983; Storey, 2008). Therefore, the presence of glycolytic enzymes in epididymal fluid can reflect spermatozoa maturation processes during epididymal transit.

The GMB pathway was indicated in bull epididymal fluid for the first time. Enzymes participating in GMB pathway can synthesize nucleotide sugar GDP- $\alpha-D-$ mannose, which can then be further metabolized to GDP-L-fucose. The GDP-L-fucose is a substrate for the construction of fucosylated glycans, which in mammals play important roles in selectin-mediated leukocyteendothelial adhesion, host-microbe interactions, and various ontogenic events (Becker and Lowe, 2003). It is noteworthy that bovine spermatozoa surface proteins have the ability to bind mannose and fucose (Defaus et al., 2016), suggesting that if the monosaccharides produced in the GMB pathway are present in epididymal fluid, it is possible that they might bind to and affect spermatozoa.

Protection of spermatozoa and reproductive tract tissues against oxidative damage is based on enzymes capable of scavenging reactive oxygen species (Williams and Ford, 2004). Both the enzyme glutathione peroxidase (GPX) and PP pathways were present in epididymal fluid. The PP pathway generates NADPH, which supplies the activity of GPX. To our knowledge, the activity of GPX and the PP pathway in the bull epididymis have so far not been described. Because PP pathway and GPX activity are well-established reactive oxygen species scavenging systems inside bull spermatozoa (Williams and Ford, 2004), the PP pathway and GPX seem to serve as anti-oxidative protection both from outside as well as inside spermatozoa cells.

Anti-oxidative protection of epididymis and spermatozoa can also be potentially exerted by the nuclear factor-erythroid 2-related factor 2 (NRF2)-mediated oxidative stress response (NOSR) pathway. This pathway is part of the cellular defense response to oxidative stress and is based NRF2 binding to the antioxidant response elements within the promoter of detoxifying and antioxidant enzymes, which activates their transcription (Nguyen et al., 2009). We found multiple proteins in epididymal fluid that are transactivated in response to oxidative stress by the NOSR pathway, including antioxidant proteins peroxiredoxin 1 and superoxide dismutase, chaperone proteins stress-inducedphosphoprotein 1, T-complex protein 1 subunit eta and peptidyl-prolyl cis-trans isomerase B precursor, and ubiquitination protein transitional endoplasmic reticulum ATPase. Because NRF2 was recently found in epi- didymal tissue of rats and humans (Wajda et al., 2016), we hypothesized that the NOSR pathway takes place in epididymal cells in response to oxidative stress, which causes an increase of previously mentioned detoxifying and antioxidant enzymes in the epididymal fluid, to protect epididymal tissues as well as spermatozoa.

Ubiquitination of bovine sperm probably serves as part of a sperm quality control system, where ubiquitinated defective spermatozoa are removed (Sutovsky et al., 2001), along with ubiquitinated cytoplasmic droplets (Temple-Smith, 1984; Kuster et al., 2004; Cooper, 2011). Epididymal fluid has been shown to possess extracellular activity of the ubiquitin system, which can take part in defective spermatozoa removal (Baska et al., 2008). Our results support the results of these authors, indicating that this activity consists of ubiquitin enzymes E1, E2, and ubiquitin carboxyl-terminal hydrolase isozyme L1 in epididymal fluid. Moreover, we have identified multiple new proteins in bull epididymal fluid that we were able to contextualize as proteins taking part in a ubiquitination pathway. Those proteins include heat shock proteins (HSP90AA1, HSP90B1), proteasome system elements (PSMA1, PSMC2, PSMC5, PSMC6, PSMD3, PSMD13), and ubiquitination enzymes (UBA1) that may play a part in epididymal sperm quality control.

The acute phase response is a nonspecific earlydefense immune system activated by trauma, infection, stress, and inflammation (Cray et al., 2009). Our results indicated the presence of acute phase response signaling proteins in bull epididymal fluid. The majority of identified proteins were proteins expressed in response to a stimulus and secreted into the extracellular space. We believe that the presence of those proteins in epididymal fluid shows the potential activity of immunological cells located in the epididymis, and suggests the acute phase response as a potential topic for future studies that would further the understanding of the immunological system activity in the epididymis.

Mammalian spermatozoa maturation in the epididymis is related to the remodeling of both protein and lipid content of spermatozoa membranes (Nikolopoulou et al., 1985; Hall et al., 1991). Two pathways that we indicated in bull epididymal fluid seem to be connected to membrane lipid reorganization: liver X receptor $(\mathbf{L X R})$ /farnesoid X receptor (FXR) and FXR/ retinoid $\mathbf{X}$ receptor ( $\mathbf{R X R}$ ) activation pathways. The LXR/FXR pathway is involved in lipid metabolism, cholesterol catabolism, and inflammation (Calkin and Tontonoz, 2012). The FXR/RXR pathway takes part in lipid and glucose homeostasis, regulation of bile acid, and inflammatory response (Ding et al., 2015). Results of Ouvrier et al. (2009) showed LXR receptors to be 


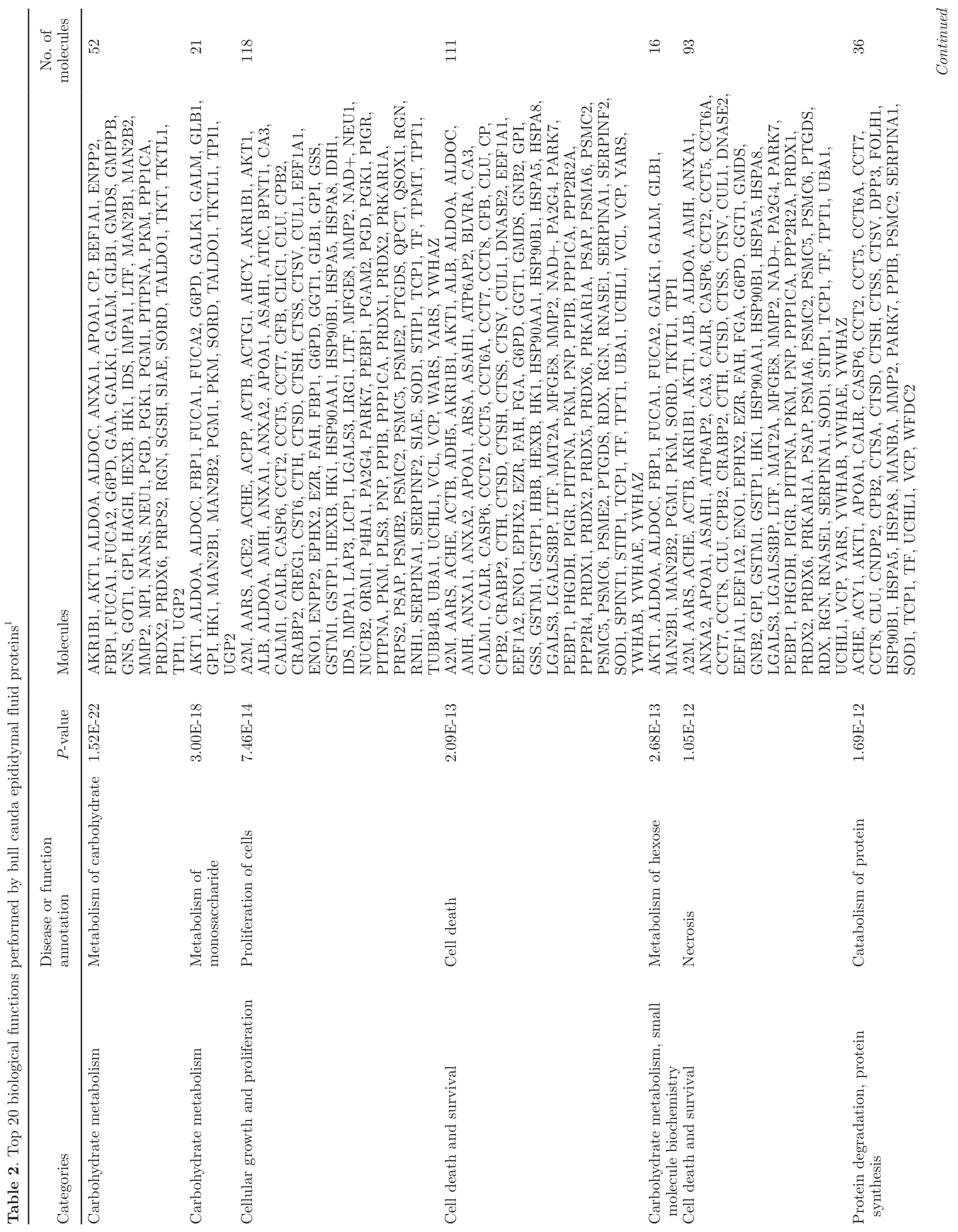




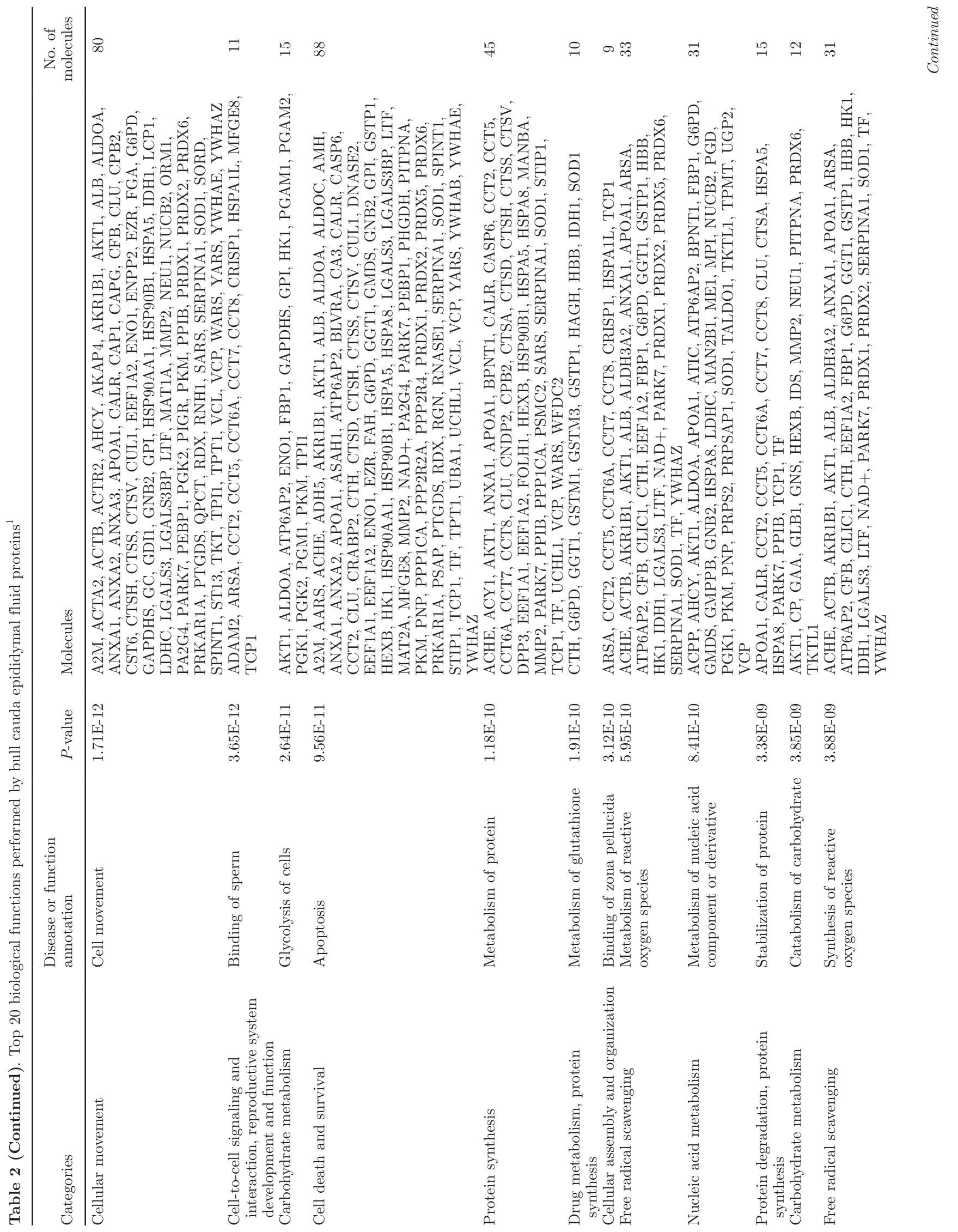




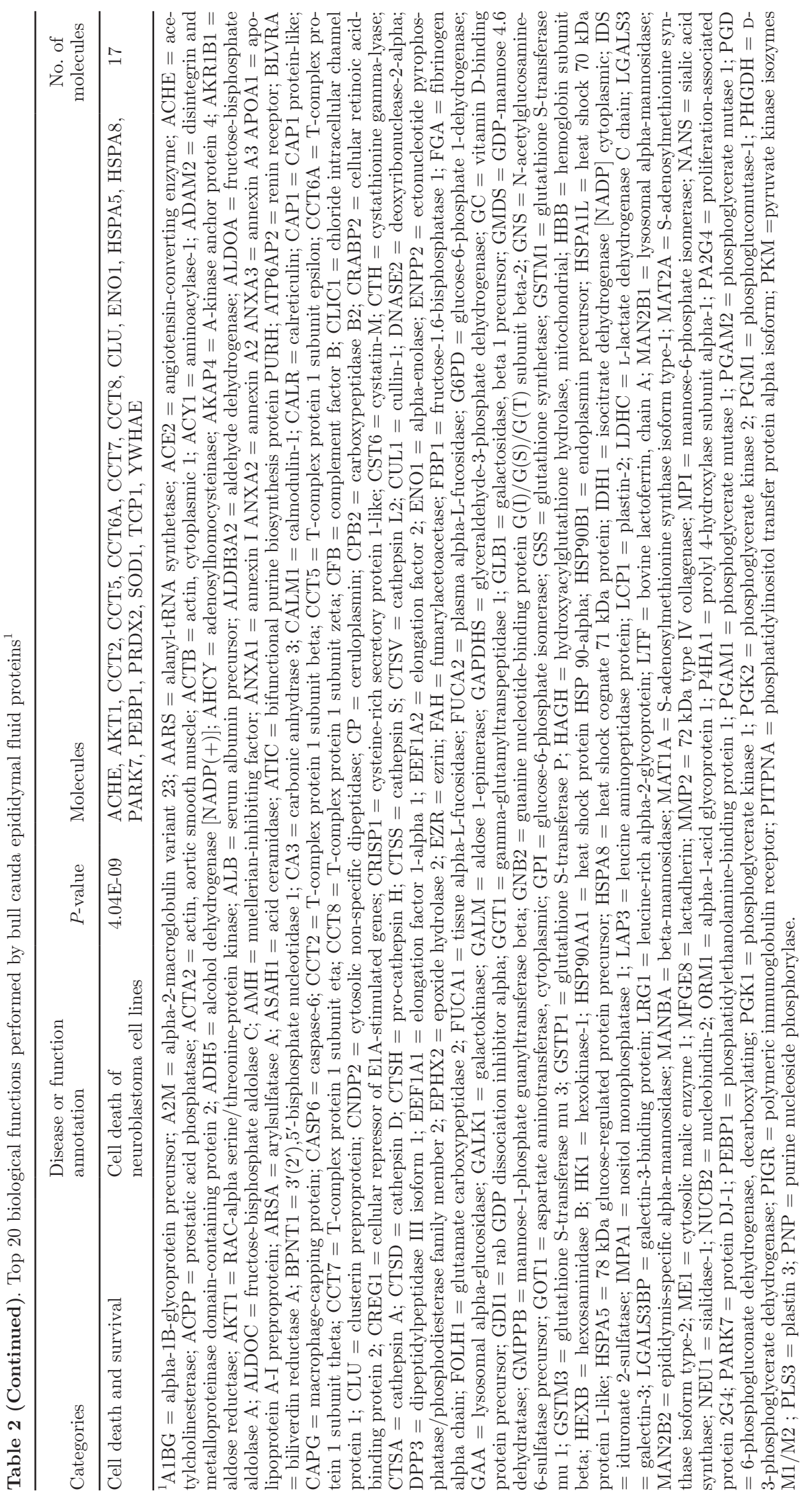


one of the key actors in cholesterol homeostasis control in mouse proximal epididymis, and this homeostasis should be investigated in the bull epididymis in future studies.

Maturation of spermatozoa is closely connected with the development of spermatozoa motility (Dacheux et al., 2012). The distinction of cellular movement category in biological function analysis strongly un- derlines this function, as it encompasses 80 proteins possibly involved in this function. The results of our research also support the idea that epididymal spermatozoa start gaining the capacity of zona binding during epididymal transit. This is evidenced by 9 proteins categorized as being involved in zona pellucida binding and 11 proteins categorized as being involved in sperm binding.
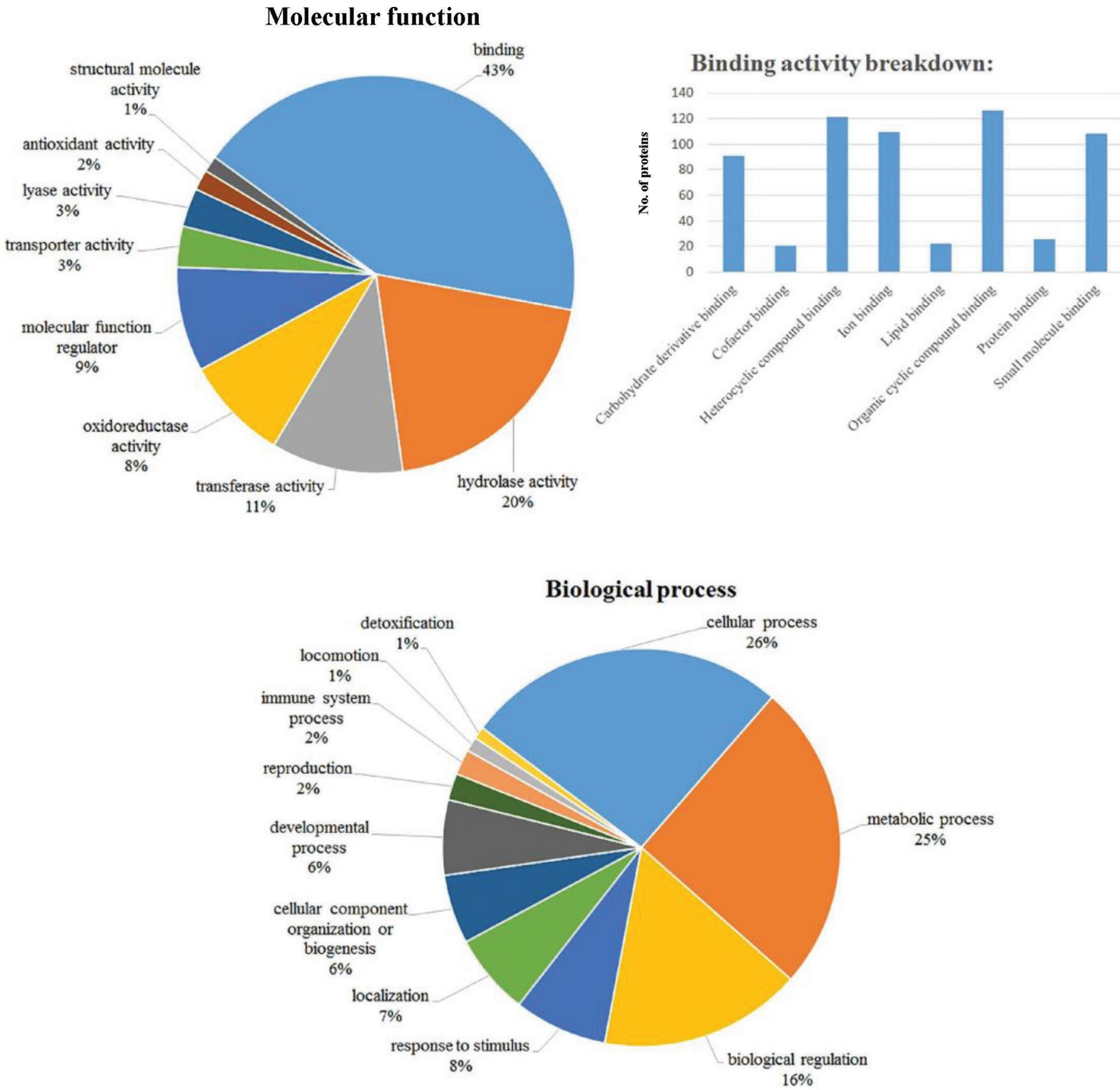

Figure 3. Ontological analysis of identified proteins from bull cauda epididymal fluid. Classification of the protein set was performed according to the Gene Ontology molecular function and biological process terms. Color version available online. 


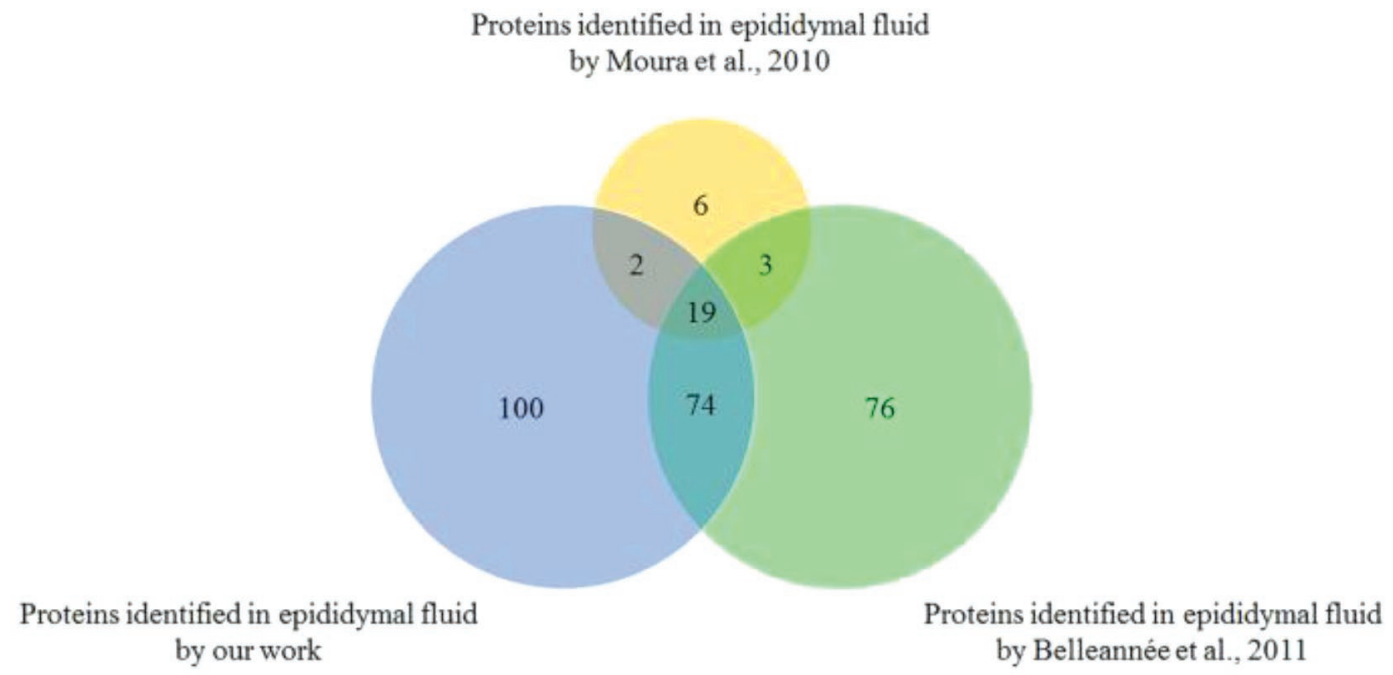

Figure 4. Venn diagram comparing the number of identified bull epididymal fluid proteins by our work, the work of Belleannée et al. (2011), and the work of Moura et al. (2010). Color version available online.

The remaining top canonical pathways indicated by IPA are mostly connected to intracellular processes. Pathways such as remodeling of epithelial adherens junctions pathway, and epithelial adherens junction signaling pathway were established mainly on the basis of actin and tubulin presence in epididymal fluid. Both those proteins are mainly intracellular. The presence of those proteins may be the result of protein leakage from damaged and removed spermatozoa, and from cytoplasmic droplets. Some of the intracellular pathways can also reflect the occurrence of processes taking place in earlier parts of the male reproductive system, such as the Sertoli cell-Sertoli cell junction signaling pathway, which occurs in the testes. This suggestion is also supported by the presence of the large protein group involved in cell proliferation, which should be very limited in epididymis, but is very robust in testes during spermatogenesis.

Proteins identified in this study have been previously reported in applied research related to cattle breeding. Glyceraldehyde-3-phosphate dehydrogenase and tubulin were found to be enriched in sexed bovine semen (De Canio et al., 2014). Alpha-enolase and isocitrate enolase have been named as having a potential role in bull fertility (Soggiu et al., 2013). Our results indicate that these proteins can exert their functions already in the epididymis. Environmental conditions prevailing in the epididymis allow storage of high concentrations of spermatozoa, and mimicking these conditions is still a challenge for in vitro storage (De Pauw et al., 2003). Reyes-Moreno et al. (2002) identified epididymal factors that protect ejaculated bovine sperm during in vitro storage as proteins, including clusterin and fibrinogen, which were identified in our study. Our results provide a comprehensive list of epididymal proteins, which should be tested in future studies regarding their sperm protection potential during in vitro storage.

Overall, our results have pointed out multiple novel pathways in bull epididymal fluid that might take part in various aspects of maturation and protection processes of epididymal spermatozoa. Carbohydrate metabolism seems to be very robust in bull epididymal fluid. The PP pathway, GPX activity, and possibly the NOSR pathway are possibly a major part of antioxidative activity in the epididymis. We also identified multiple new proteins taking part in extracellular ubiquitination processes and in phagocytosis, both of which could be involved in epididymal sperm quality control. We believe that we have indicated new areas that could be targeted in future research to better elucidate the complex functions and interactions of the bull epididymal fluid proteome.

\section{ACKNOWLEDGMENTS}

This work was supported by grants 2013/09/N/ NZ9/01655 and 2013/09/B/NZ9/01752 from the National Science Center (Kraków, Poland) and funds appropriated to the Institute of Animal Reproduction and Food Research. We thank the personnel of the Breeding and Insemination Station in Karczew (Poland) for providing the bull epididymal fluid. The article processing charge was covered by the KNOW Consortium: "Healthy Animal-Safe Food" (Ministry of Sciences and Higher Education; Dec: 05-1/KNOW2/2015). 


\section{REFERENCES}

Baska, K. M., G. Manandhar, D. Feng, Y. Agca, M. W. Tengowski, M. Sutovsky, Y. J. Yi, and P. Sutovsky. 2008. Mechanism of extracellular ubiquitination in the mammalian epididymis. J. Cell. Physiol. 215:684-696.

Becker, D. J., and J. B. Lowe. 2003. Fucose: Biosynthesis and biological function in mammals. Glycobiology $13: 41 \mathrm{R}-53 \mathrm{R}$

Belleannée, C., V. Labas, A. P. Teixeira-Gomes, J. L. Gatti, J. L. Dacheux, and F. Dacheux. 2011. Identification of luminal and secreted proteins in bull epididymis. J. Proteomics 74:59-78.

Calkin, A. C., and P. Tontonoz. 2012. Transcriptional integration of metabolism by the nuclear sterol-activated receptors LXR and FXR. Nat. Rev. Mol. Cell Biol. 13:213-224.

Cooper, T. G. 2011. The epididymis, cytoplasmic droplets and male fertility. Asian J. Androl. 13:130-138.

Cray, C., J. Zaias, and N. H. Altman. 2009. Acute phase response in animals: A review. Comp. Med. 59:517-526.

D'Amours, O., G. Frenette, M. Fortier, P. Leclerc, and R. Sullivan. 2010. Proteomic comparison of detergent-extracted sperm proteins from bulls with different fertility indexes. Reproduction 139:545556.

Dacheux, J. L., C. Belleannée, B. Guyonnet, V. Labas, A. P. Teixeira-Gomes, H. Ecroyd, X. Druart, J. L. Gatti, and F. Dacheux. 2012. The contribution of proteomics to understanding epididymal maturation of mammalian spermatozoa. Syst Biol Reprod Med 58:197-210.

Dacheux, J. L., F. Dacheux, and X. Druart. 2016. Epididymal protein markers and fertility. Anim. Reprod. Sci. 169:76-87.

De Canio, M., A. Soggiu, C. Piras, L. Bonizzi, A. Galli, A. Urbani, and P. Roncada. 2014. Differential protein profile in sexed bovine semen: Shotgun proteomics investigation. Mol. Biosyst. 10:12641271.

Defaus, S., M. Avilés, D. Andreu, and R. Gutiérrez-Gallego. 2016. Identification of bovine sperm surface proteins involved in carbohydrate-mediated fertilization interactions. Mol. Cell Proteomics 15:2236-2251.

De Pauw, I.M.C., A. Van Soom, K. Mintiens, S. Verberckmoes, and A. de Kruif. 2003. In vitro survival of bovine spermatozoa stored at room temperature under epididymal conditions. Theriogenology 59:1093-1107.

Ding, L., L. Yang, Z. Wang, and W. Huang. 2015. Bile acid nuclear receptor FXR and digestive system diseases. Acta Pharmacol. Sin. B 5:135-144.

Hall, J. C., J. Hadley, and T. Doman. 1991. Correlation between changes in rat sperm membrane lipids, protein, and the membrane physical state during epididymal maturation. J. Androl. 12:76-87.

Hoskins, D. D., D. Munsterman, and M. L. Hall. 1975. The control of bovine sperm glycolysis during epididymal transit. Biol. Reprod. 12:566-572.

Huntley, R. P., T. Sawford, P. Mutowo-Muellenet, A. Shypitsyna, C. Bonilla, M. J. Martin, and C. O'Donovan. 2015. The GOA database: Gene Ontology annotation updates for 2015. Nucleic Acids Res. 43:D1057-D1063.

Juyena, N. S., and C. Stelletta. 2012. Seminal plasma: An essential attribute to spermatozoa. J. Androl. 33:536-551.

Kelly, V. C., S. Kuy, D. J. Palmer, Z. Xu, S. R. Davis, and G. J. Cooper. 2006. Characterization of bovine seminal plasma by proteomics. Proteomics 6:5826-5833.

Kuster, C. E., R. A. Hess, and G. C. Althouse. 2004. Immunofluorescence reveals ubiquitination of retained distal cytoplasmic droplets on ejaculated porcine spermatozoa. J. Androl. 25:340-347.

Moura, A. A., D. A. Chapman, H. Koc, and G. J. Killian. 2006. Proteins of the cauda epididymal fluid associated with fertility of mature dairy bulls. J. Androl. 27:534-541.
Moura, A. A., D. A. Chapman, H. Koc, and G. J. Killian. 2007. A comprehensive proteomic analysis of the accessory sex gland fluid from mature Holstein bulls. Anim. Reprod. Sci. 98:169-188.

Moura, A. A., C. E. Souza, B. A. Stanley, D. A. Chapman, and G. J. Killian. 2010. Proteomics of cauda epididymal fluid from mature Holstein bulls. J. Proteomics 73:2006-2020.

Nguyen, T., P. Nioi, and C. B. Pickett. 2009. The Nrf2-antioxidant response element signaling pathway and its activation by oxidative stress. J. Biol. Chem. 284:13291-13295.

Nikolopoulou, M., A. S. Donald, and J. C. Vary. 1985. Changes in the lipid content of boar sperm plasma membranes during epididymal maturation. Biochim. Biophys. Acta 815:486-498.

Odhiambo, J. F., and R. A. Dailey. 2011. Characterization of proteins in cryopreserved and non-cryopreserved seminal plasma of dairy bulls of differing fertility. Open J. Anim. Sci. 1:33-40.

Ouvrier, A., R. Cadet, P. Vernet, B. Laillet, J. M. Chardigny, J. M. A. Lobaccaro, J. R. Drevet, and F. Saez. 2009. LXR and ABCA1 control cholesterol homeostasis in the proximal mouse epididymis in a cell-specific manner. J. Lipid Res. 50:1766-1775.

Panse, G. T., S. Jayaraman, and A. R. Sheth. 1983. Shift of glycolysis as a marker of sperm maturation. Arch. Androl. 11:137-140.

Perkins, D. N., D. J. C. Pappin, D. M. Creasy, and J. S. Cottrell. 1999. Probability-based protein identification by searching sequence databases using mass spectrometry data. Electrophoresis 20:3551-3567.

Reyes-Moreno, C., M. Boilard, R. Sullivan, and M. A. Sirard. 2002. Characterization and identification of epididymal factors that protect ejaculated bovine sperm during in vitro storage. Biol. Reprod. $66: 159-166$.

Robaire B., B. T. Hinton, and M. C. Orgebin-Crist. 2006. The epididymis. Pages 1071-1148 in Knobil and Neill's Physiology of Reproduction. 3rd ed. Elsevier, Amsterdam, the Netherlands.

Soggiu, A., C. Piras, H. A. Hussein, M. De Canio, A. Gaviraghi, A. Galli, A. Urbani, L. Bonizzia, and P. Roncada. 2013. Unravelling the bull fertility proteome. Mol. Biosyst. 9:1188-1195.

Storey, B. T. 2008. Mammalian sperm metabolism: Oxygen and sugar, friend and foe. Int. J. Dev. Biol. 52:427-437.

Sutovsky, P., R. Moreno, J. Ramalho-Santos, T. Dominko, W. E. Thompson, and G. Schatten. 2001. A putative, ubiquitin-dependent mechanism for the recognition and elimination of defective spermatozoa in the mammalian epididymis. J. Cell Sci. 114:16651675 .

Temple-Smith, P. D. 1984. Phagocytosis of sperm cytoplasmic droplets by a specialized region in the epididymis of the brushtailed possum, Trichosurus vulpecula. Biol. Reprod. 30:707-720.

Wajda, A., J. Łapczuk, M. Grabowska, M. Słojewski, M. Laszczyńska, E. Urasińska, and M. Droździk. 2016. Nuclear factor E2-related factor-2 (Nrf2) expression and regulation in male reproductive tract. Pharmacol. Rep. 68:101-108.

Westfalewicz, B., M. A. Dietrich, A. Mostek, A. Partyka, W. Bielas, W. Niżański, and A. Ciereszko. 2017. Analysis of bull (Bos taurus) seminal vesicle fluid proteome in relation to seminal plasma proteome. J. Dairy Sci. 100:2282-2298.

Wilkins, M. R., J. Sanchez, A. A. Gooley, R. D. Appel, I. HumpherySmith, D. F. Hochstrasser, and K. L. Williams. 1996. Progress with proteome projects: Why all proteins expressed by a genome should be identified and how to do it. Biotechnol. Genet. Eng. Rev. 13:19-50.

Williams, A. C., and W. C. L. Ford. 2004. Functional significance of the pentose phosphate pathway and glutathione reductase in the antioxidant defenses of human sperm. Biol. Reprod. 71:1309-1316. 